\section{PATIENT SAFETY}

A.-L. Manninen

J.-M. Isokangas

A. Karttunen

T. Siniluoto

M.T. Nieminen

\title{
A Comparison of Radiation Exposure between Diagnostic CTA and DSA Examinations of Cerebral and Cervicocerebral Vessels
}

\begin{abstract}
BACKGROUND AND PURPOSE: While the number of CTA examinations is continually increasing compared with DSA examinations, there is little comparative dose information about the different imaging techniques. We compared patient radiation exposure resulting from diagnostic CTA and DSA examinations for both cerebral and cervicocerebral vessels.
\end{abstract}

\begin{abstract}
MATERIALS AND METHODS: An anthropomorphic phantom was irradiated by using typical diagnostic CTA and DSA setups and imaging parameters. For both imaging techniques, the imaging area of cerebral vessels included intracranial vessels only, while the imaging area of cervicocerebral vessels included both cervical and intracranial vessels from the aortic arch to the vertex. The effective dose was determined by using RPLDs. The DSA examination was simulated by using a biplane angiography system, and the CTA examination, by using a 64-row multidetector CT scanner.
\end{abstract}

RESULTS: For the imaging of cerebral vessels, the effective dose according to ICRP 103 was $0.67 \mathrm{mSv}$ for CTA and $2.71 \mathrm{mSv}$ for DSA. For the imaging of cervicocerebral vessels, the effective dose was 4.85 $\mathrm{mSv}$ for CTA and $3.60 \mathrm{mSv}$ for DSA. The maximum absorbed dose (milligray) for skin, brain, salivary glands, and eyes was 166.2, 73.5, 35.6, and $21.8 \mathrm{mGy}$ for DSA and 19.0, 16.9, 20.4, and $14.8 \mathrm{mGy}$ for CTA, respectively. The conversion factors from DAP and DLP to effective dose were calculated.

concLUSIONS: The effective dose for CTA assessment of cerebral vessels was approximately onefifth the dose compared with DSA. In the imaging of cervicocerebral vessels, the effective dose for CTA was approximately one-third higher compared with DSA.

ABBREVIATIONS: $\mathrm{AEC}=$ automatic exposure control; $\mathrm{CV}=$ coefficient of variation; $\mathrm{DAP}=$ dosearea product, DLP = dose-length product; ICRP = International Commission on Radiological Protection; $\mathrm{RPL}=$ radiophotoluminescence; $\mathrm{RPLD}=$ radiophotoluminescence dosimeter

$\mathbf{T}$

he combination of 3D rotational and 2D DSA produces

high-resolution and high-contrast images of the cervical and cerebral vessels. ${ }^{1-3}$ Multidetector CTA also permits rapid scanning with a narrow section thickness and isotropic voxel data with superior resolution in the $\mathrm{z}$-axis to evaluate cerebral and cervical vascular pathologies. ${ }^{1,4}$ An important principle guiding the diagnostic use of radiation is to keep the radiation exposure as low as reasonably achievable. Because DSA and CTA have very different irradiation geometries and the radiation exposure is reported in different quantities, the effective dose is a useful means of comparing the stochastic risks, such as cancer induction, malignant disease or heritable effects caused by an exposure to ionization radiation. ${ }^{5}$ The absorbed doses of organs predict the deterministic risks such as a temporary epilation, hair loss, or opacity of the lens. ${ }^{6-9}$

Previous investigations have indicated a large variation between radiation exposures for cervical and cerebral angiogra-

Received December 31, 2011; accepted after revision February 25, 2012.

From the Department of Diagnostic Radiology (A.-L.M., J.-M.I., A.K., T.S., M.T.N.), Oulu University Hospital, Oulu, Finland; and Department of Medical Technology (M.T.N.), University of Oulu, Oulu, Finland.

Please address correspondence to Anna-Leena Manninen, MSc, Department of Diagnostic Radiology, Box 50, FIN-90029, Oulu University Hospital, Oulu, Finland; e-mail: Anna-Leena. Manninen@ppshp.fi

Indicates article with supplemental on-line tables

Indicates article with supplemental on-line figure.

http://dx.doi.org/10.3174/ajnr.A3123 phy, depending on the imaged area and the imaging technique used. ${ }^{2,3,10-14} \mathrm{~A}$ comparison of the radiation exposure between CTA and DSA methods has not been previously performed by using the same dose-measurement setup, to our knowledge. Also, the lack of reliable conversion factors for angiographic examinations complicates the comparison between the different imaging methods.

In the present study, our aim was to objectively compare the radiation exposure of CTA and DSA, by using the same dose-determination technique. The radiation exposure was determined for both imaging techniques for a typical 4-vessel angiography of the cerebral area, including intracranial vessels, and for the cervicocerebral area, including both cervical and intracranial vessels. The determination of the organ and effective doses of the patients was based on phantom dose measurements with RPLDs. Conversion factors from the DAP and the DLP to the effective dose were calculated.

\section{Materials and Methods}

\section{Imaging Equipment and Protocols}

CTA was performed by using a 64-row multidetector CT scanner (Somatom Sensation 64; Siemens, Erlangen, Germany). For DSA, we used biplane angiography equipment (Integris Allura 12 and 15 biplane; Philips Healthcare, Best, the Netherlands). To determine organ and effective doses, we used diagnostically optimized protocols of CTA and DSA for cerebral (intracranial) and cervicocerebral (from 
Table 1: Technical parameters for different CTA examinations

\begin{tabular}{lcc}
\hline Scan Parameters & $\begin{array}{c}\text { Protocol 1: } \\
\text { Cerebral Scan with AEC }\end{array}$ & $\begin{array}{c}\text { Protocol 2: } \\
\text { Cervicocerebral Scan without AEC }\end{array}$ \\
\hline Topogram & $120 \mathrm{kV}(\mathrm{p}), 35 \mathrm{mAs}$ & $120 \mathrm{kV}(\mathrm{p}), 60 \mathrm{mAs}$ \\
Premonitoring $^{\mathrm{a}}$ & $120 \mathrm{kV}(\mathrm{p}), 40 \mathrm{mAs}$ & $120 \mathrm{kV}(\mathrm{p}), 40 \mathrm{mAs}$ \\
Monitoring $^{\mathrm{b}}$ & $120 \mathrm{kV}(\mathrm{p}), 40 \mathrm{mAs}$ & $120 \mathrm{kV}(\mathrm{p}), 40 \mathrm{mAs}$ \\
Tube voltage $(\mathrm{kV}[\mathrm{p}])_{\text {Effective mAs/quality ref. mAs }}$ & 100 & 120 \\
Pitch factor & $148 / 160$ & 160 (constant tube current) \\
Rotation time $(\mathrm{sec})$ & 1.20 & 1.20 \\
Acquisition & 0.5 & 0.33 \\
Slice collimation $(\mathrm{mm})$ & $64 \times 0.6 \mathrm{~mm}$ & $64 \times 0.6 \mathrm{~mm}$ \\
Section width $(\mathrm{mm})$ & 0.6 & 0.6 \\
Scan range $(\mathrm{cm})$ & 4.0 & 5.0
\end{tabular}

a One section scanned at the level of aortic arch.

${ }^{b}$ Five sections scanned at the level of aortic arch.

Table 2: Technical parameters for different DSA examinations; protocol 1: diagnostic angiography of cerebral vessels ${ }^{\mathrm{a}}$

\begin{tabular}{|c|c|c|c|c|c|c|c|}
\hline Vessels & RICA Head & RICA Head & RICA Head & RICA Head & RVA Head & LICA Head & LVA Head \\
\hline \multirow{3}{*}{$\begin{array}{l}\text { Projections for DSA runs } \\
\text { fps:duration }\end{array}$} & AP/LAT biplane & Oblique monoplane & Oblique monoplane & 3D RA & AP /LAT biplane & AP/LAT biplane & AP/LAT biplane \\
\hline & $2 \mathrm{fps}: 4 \mathrm{~s}$ & 2 fps:4 s & $2 \mathrm{fps}: 4 \mathrm{~s}$ & $25 \mathrm{fps}: 4 \mathrm{~s}$ & 2 fps:4 s & $2 \mathrm{fps}: 4 \mathrm{~s}$ & 2 fps:4 s \\
\hline & 1 fps:5 s & 1 fps: $2 \mathrm{~s}$ & $1 \mathrm{fps}: 2 \mathrm{~s}$ & & $1 \mathrm{fps}: 5 \mathrm{~s}$ & $1 \mathrm{fps}: 5 \mathrm{~s}$ & $1 \mathrm{fps}: 5 \mathrm{~s}$ \\
\hline No. of frames & 26 & 10 & 10 & 100 & 26 & 26 & 26 \\
\hline FOV $(\mathrm{cm})$ & $22 / 23$ & 17 & 17 & 17 & $22 / 23$ & $22 / 23$ & $22 / 23$ \\
\hline SID & $102 / 99$ & 102 & 103 & 119 & $104 / 99$ & $106 / 100$ & 102/101 \\
\hline kV(p) (AP/LAT) & $80 / 75$ & $85 /-$ & $82 /-$ & $92 /-$ & $80 / 75$ & $80 / 75$ & $80 / 75$ \\
\hline$m A s$ per $f(A P / L A T)$ & $91 / 25$ & $102 /-$ & $101 /-$ & $1.1 /-$ & $114 / 28$ & $97 / 25$ & $92 / 27$ \\
\hline
\end{tabular}

Note:-RICA indicates right internal carotid artery; LICA, left internal carotid artery; RVA, right internal vertebral artery; LVA, left internal vertebral artery; 3D RA, 3D rotational angiography; $A P$, anterior posterior direction; LAT, lateral direction; fps, frames per second; SID, source to image distance (cm); mAs, tube current time product; Al, aluminum, HVL, half-value layer;

Cu, copper.
a AEC was used. Filtration was $2.9 \mathrm{~mm} \mathrm{Al} \mathrm{HVL}$ and $0.1 \mathrm{~mm} \mathrm{Cu}$. The total number of frames was 224 in protocol 1 and 275 in protocol 2 . The fluoroscopy time was 5 minutes in both protocols.

\begin{tabular}{|c|c|c|c|c|c|c|c|c|c|c|c|}
\hline Vessels & $\begin{array}{l}\text { Aortic } \\
\text { Arch }\end{array}$ & $\begin{array}{l}\text { RICA } \\
\text { Neck }\end{array}$ & $\begin{array}{l}\text { RICA } \\
\text { Head }\end{array}$ & $\begin{array}{l}\text { RICA } \\
\text { Neck }\end{array}$ & $\begin{array}{l}\text { RICA } \\
\text { Neck }\end{array}$ & RVA Neck & $\begin{array}{l}\text { RVA } \\
\text { Head }\end{array}$ & LICA/Neck & $\begin{array}{l}\text { LICA } \\
\text { Head }\end{array}$ & LVA Neck & $\begin{array}{l}\text { LVA } \\
\text { Head }\end{array}$ \\
\hline Projections & $\mathrm{AP}$ & $\begin{array}{r}\text { Obliq. LAT } \\
\text { biplane }^{\text {b }}\end{array}$ & $\begin{array}{l}\text { AP/LAT } \\
\text { biplane }^{\mathrm{b}}\end{array}$ & 3DRA & $\begin{array}{l}\text { Obliq. } \\
\text { monoplane }\end{array}$ & $\begin{array}{l}\text { Obliq. } \\
\text { monoplane }\end{array}$ & $\begin{array}{l}\text { AP/LAT } \\
\text { biplane }^{\text {b }}\end{array}$ & $\begin{array}{l}\text { Obliq. LAT } \\
\text { biplane }^{\mathrm{b}}\end{array}$ & $\begin{array}{l}\text { AP/LAT } \\
\text { biplane }^{b}\end{array}$ & $\begin{array}{l}\text { Obliq. } \\
\text { monoplane }\end{array}$ & $\begin{array}{l}\text { AP/LAT } \\
\text { biplane }\end{array}$ \\
\hline fps & 1 & 2 and 1 & 2 and 1 & 25 & 1 & 1 & 2 and 1 & 2 and 1 & 2 and 1 & 1 & 2 and 1 \\
\hline Duration(s) & 15 & 3 and 1 & 3 and 7 & 4 & 10 & 9 & 3 and 7 & 3 and 1 & 3 and 7 & 9 & 3 and 7 \\
\hline No. of frames & 15 & 14 & 26 & 100 & 10 & 9 & 26 & 14 & 26 & 9 & 26 \\
\hline FOV $(\mathrm{cm})$ & 30 & $22 / 23$ & $22 / 23$ & 17 & 17 & 30 & $22 / 23$ & $22 / 23$ & $22 / 23$ & 30 & $22 / 23$ \\
\hline $\begin{array}{l}\text { SID } \\
\text { (AP/LAT) }\end{array}$ & 104 & $105 / 107$ & $102 / 99$ & 119 & 103 & 105 & $104 / 99$ & $105 / 104$ & $106 / 100$ & 104 & $102 / 101$ \\
\hline kV(p) (AP/LAT) & $70 /-$ & $80 / 75$ & $80 / 75$ & $75 /-$ & 80/- & $79 /-$ & $80 / 75$ & $80 / 75$ & $80 / 75$ & $80 /-$ & $80 / 75$ \\
\hline $\mathrm{mAs} / \mathrm{f}(\mathrm{AP} / \mathrm{LAT})$ & $31 /-$ & $20 / 16$ & $73 / 24$ & $1.0 /-$ & $31 /-$ & $14 /-$ & $71 / 26$ & $26 / 15$ & $71 / 25$ & $20 /-$ & $71 / 27$ \\
\hline
\end{tabular}

Note:-Obliq. Indicates oblique; RICA, right internal carotid artery; LICA, left internal carotid artery; RVA, right internal vertebral artery; LVA, left internal vertebral artery, 3D RA, 3D rotational angiography; $A P$, anterior posterior direction; LAT, lateral direction; fps, frames per second; SID, source to image distance (cm); mAs, tube current time product; Al, aluminum; HVL, half-value layer; 'Cu, copper.

a AEC was used. Filtration was 2.9-mm AI HVL and 0.1-mm Cu. The total number of frames was 224 in protocol 1 and 275 in protocol 2 . The fluoroscopy time was 5 minutes in both protocols.

b The imaging is shown in 2 parts: fast-phase and slow-phase.

the aortic arch to vertex) vessels (Tables $1-3$ ). The imaging of the cerebral vessels began at phantom level 5 , and the cervicocerebral vessels, at phantom level 15 (Fig 1). For CTA, a protocol with AEC was used when imaging the cerebral vessels. AEC was not used for imaging cervicocerebral vessels, to maintain adequate image quality throughout the imaging area. The overeffective tube-current modulation in the head area in this AEC implementation reduces sensitivity in detecting small aneurysms or small arteries. Therefore, the CTA protocol with constant tube current is routinely used for examination of cervicocerebral vessels at our institution. The optimization was done to prevent false-negative and confusing results, which may evoke another angiographic examination, such as DSA. The DSA protocols were also optimized by a neuroradiologist and are in routine clinical use at our institution.

\section{Dose Measurement}

Dose measurements were performed by simulating CTA and DSA acquisitions with an anthropomorphic phantom (Alderson-Rando Phantom; Alderson Research Laboratories, Long Island City, New York) consisting of a human skeleton and simulated lung tissue with similar attenuation as that of lung, encased in tissue-equivalent plastic material. The phantom is molded to the shape of the human body and divided into transverse sections of $2.5-\mathrm{cm}$ thickness, including holes in the z-direction to place the long axis of the dosimeters perpendic- 


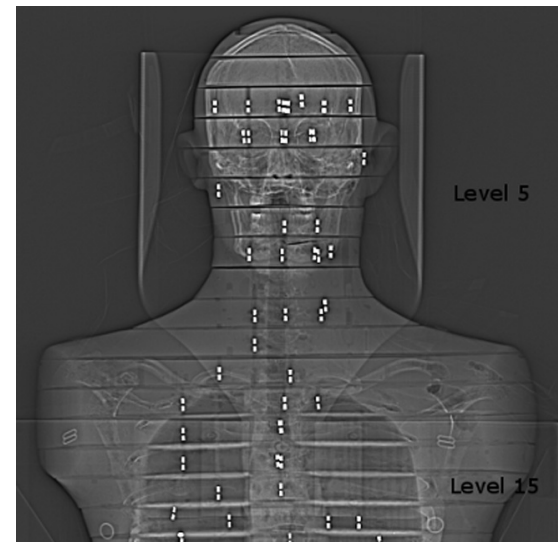

Fig 1. The imaging of the cerebral vessels began at phantom level 5 , and the cervicocerebral vessels, at phantom level 15. RPL dosimeters are shown as bright rods in the scout image.

ular to the photon beam. Simulated breast tissue is made of paraffin with similar attenuation as fat. RPLDs were implanted in each organ as previously published. ${ }^{15}$ All organs within the primary beam were covered by the dosimeters. Dose measurements were also performed for organs outside the primary beam because these are exposed to scattered radiation.

Depending on the organ volume, 1-9 dosimeters were placed in the organ in the same positions with both imaging methods. Also, depending on the acquisition protocol, the phantom was equipped with at least 69 RPL dosimeters (On-line Table 1 and On-line Fig 1). Three simulation runs were performed for both CTA and DSA procedures. The phantom was adjusted on the CT imaging table by using laser light. Each CTA scan was rerun 3 times, and the absorbed dose recorded by the dosimeters was divided by 3 to give the result of 1 scan. Laser light positioning was not available for DSA, which could cause some uncertainty in the different simulation runs. The mean dose of the dosimeters represents the organ dose. The equivalent doses for the tissues, such as active bone marrow, bone surface, colon, skin, extra thoracic airways, lymph nodes, and muscles, were calculated in accordance with previous publications. ${ }^{16-19}$ Various tissues or organs were assigned in the definitions of the ICRP of the effective dose. The effective dose is defined as follows: $\mathrm{E}=\sum_{\mathrm{T}} W_{\mathrm{T}} \times H_{\mathrm{T}}$, where $W_{\mathrm{T}}$ is the tissue-weighting factor for both ICRP 60 and ICRP 103 definitions (On-line Table 1). $H_{\mathrm{T}}=\sum_{\mathrm{R}} W_{\mathrm{R}} \times D_{\mathrm{T}, \mathrm{R}}$, where $W_{R}$ is the radiation weighting factor and $D_{\mathrm{T}, \mathrm{R}}(\mathrm{mGy})$ is the absorbed dose measured in tissue $(\mathrm{T})$ with radiation $(\mathrm{R})$. For $\mathrm{x}$-ray photons, $W_{\mathrm{R}}=1$, so the equivalent dose is numerically equal to the mean absorbed dose in milligrays. $^{20,21}$

\section{Dosimetry Technique}

The RPLD GD-352M with a tin filter (Dose Ace, glass dosimeter; Asahi Techno Glass, Funabashi City, Japan) and the FDG-1000 reader (ATGC-2004; Asahi Techno Glass) were used for dose measurements, as described previously. ${ }^{22}$ Dose calibration for dosimeters was performed against an ionization chamber confirmed by a radiation model controller (model 9015, with model $10 \times 6-6$, General Purpose In Beam Chamber; Radial Corporation, Monrovia, California), which is calibrated once a year. The DAP was given by the DAP meter of the imaging equipment. Calibration accuracy for the RPLD, ionization chamber, and DAP meter was determined (On-line Table 2).
Measurement Uncertainties and Statistical Analysis

In this work, the standard uncertainty of individual RPL was calculated according to international recommendations ${ }^{23}$ as follows: $u\left(D_{\mathrm{i}}\right)$ $=\left(\left[u_{\mathrm{D}}\left(R_{\text {meas }}\right)\right]^{2}+\left[u_{\mathrm{D}}\left(K_{\mathrm{E}}\right)\right]^{2}\right)^{0.5}$, where $u_{\mathrm{D}}\left(R_{\text {meas }}\right)$ is the standard uncertainty of the RPL measurement and $u_{D}\left(K_{E}\right)$ is the standard uncertainty of the energy correlation factor. In this study, $u_{\mathrm{D}}\left(R_{\text {meas }}\right)=$ $6.0 \%(20 \mu \mathrm{Gy}-11 \mathrm{mGy})$ and $u_{\mathrm{D}}\left(K_{\mathrm{E}}\right)=6.0 \%(75-125 \mathrm{keV})$. Then $u\left(D_{i}\right)=8.5 \%$. Each DSA acquisition and each CTA protocol 1 were simulated 3 times. The standard uncertainty of the estimated dose $(D)$ for each measurement point was calculated by $u(D)=1 / \mathrm{N}^{0.5} \times$ $u\left(D_{\mathrm{i}}\right)$. With 3 experiments and 1 dosimeter in each measurement point, $n=3$ and $u(D)=4.9 \%$. CTA protocol 2 was simulated once: thus, $n=1$ and $u(D)=8.5 \%$.

\section{Results}

The mean equivalent doses to the organs and the effective doses are displayed in On-line Table 3. The present study showed, for cerebral vessels, that the effective dose for CTA was one-fifth the dose compared with DSA. In the imaging of the cervicocerebral vessels, the effective dose for CTA was approximately one-third higher compared with DSA.

For the assessment of cerebral vessels, the absorbed doses in the head area for DSA were 2-7 times higher than those for CTA. The absorbed doses for the thyroid, thymus, and esophagus were somewhat similar, with low doses in these regions. For the assessment of cervicocerebral vessels, the absorbed doses in the head area for DSA were 2-3 times higher than those for CTA, whereas for the thyroid, thymus, and esophagus, the doses were higher in the CTA examination. The absorbed doses for the thoracic and abdominal region were higher in CTA than in DSA examinations.

Reproducibility of the RPLD measurements indicated sufficient accuracy in diagnostic radiology. The CV of the absorbed dose measurements was $4.5 \%$ on average for CTA and $9.0 \%$ on average for DSA, respectively. However, CV\% was only $0.6 \%-3.6 \%$ for the effective-dose measurements for all examinations.

\section{Discussion}

The aim of the present study was to objectively compare the radiation exposure due to DSA versus CTA for the diagnostic assessment of cerebral and cervicocerebral vessels by using the same dose-determination technique. The effective dose facilitates the comparison of biologic effect between the diagnostic examinations of different types or those having different acquisition parameters. The absorbed dose of the organs was determined to calculate the effective dose and to obtain information relative to dose levels causing deterministic risks. To our knowledge, this is the first study to compare DSA and CTA doses in a phantom and to determine the effective dose according to the new organ-weighting factors of the ICRP.

The radiation-related stochastic risk of a diagnostic cerebral angiography examination is primarily focused on the brain and the salivary glands. ${ }^{6}$ The increased risk of benign and malignant tumors of the brain is small in comparison with the likelihood of developing malignancy in elderly patients who usually undergo such examinations. ${ }^{24}$ Nevertheless, increased cancer risk exists for children and younger patients. The estimated lifetime risk of developing radiation-related brain cancer relative to nonexposed children was increased by 
2\%-10\% when the average absorbed dose to the brain was 17 and $163 \mathrm{mGy}$, respectively. ${ }^{25}$ The prevalence of salivary gland tumors is $3 \%$ of all head and neck cancers. ${ }^{26}$ Increased cancer risk for the salivary glands has been reported after an 11-year latency period with low-dose irradiation ${ }^{27}$ and for young patients at $<20$ years after undergoing radiation therapy treatment. ${ }^{28}$ Leukemia related to radiation exposure is a consequence of the active bone marrow exposed to high radiation doses. $^{21}$ The active bone marrow of the head area accounts for $11 \%$ of the total active bone marrow ${ }^{16}$ and does not play a considerable role in relation to leukemia risk for adults. ICRP notes that the absorbed dose range up to around $100 \mathrm{mGy}$ does not generate clinically relevant functional impairment for any tissue. The threshold value for opacities of the lens is 500-2000 $\mathrm{mGy}$, and for skin epilation or temporary hair loss, 2000-3000 $\mathrm{mGy}^{21}$

Our study indicated a low stochastic risk for CTA procedures of the cerebral vessels. The effective dose is 5 times lower than that in the same examination with DSA. Similarly, the organ doses over the primary beam area in CTA were lower compared with DSA. The higher stochastic risk for a DSA procedure of the cerebral vessels was strongly dependent on higher absorbed doses in the head and neck area. The stochastic risk increases with cervicocerebral examinations, because the irradiation exceeds the areas of radiosensitive organs, which are weighted for the highest cancer risk. ${ }^{21}$ The present study showed that a CTA procedure for cervicocerebral vessels induces a $35 \%$ higher stochastic risk compared with the same examination with DSA. The absorbed doses of the thyroid, thymus, esophagus, and lungs were higher with the CTA procedure than with DSA.

The reason for this higher risk is the imaging technique and parameters used in CTA scanning for cervicocerebral vessels. The tube voltage, tube current, and scan speed must be kept high enough to acquire images with sufficient quality and to maintain image resolution comparable with that in DSA. Also, a thin acquisition-section width is necessary to avoid partial volume artifacts. ${ }^{3,29}$ The imaging parameters above result in improved spatial resolution and higher radiation exposure. While CTA provides high image quality, DSA is still generally considered the criterion standard. Differences between the image quality of DSA and CTA are explained by the vessel-tobackground ratio and are enhanced in DSA due to the subtraction technique, eliminating undesirable image information. ${ }^{3}$ The DSA technique enables the use of lower tube voltage and tube-current time product than with CTA. With DSA procedures, the number of images acquired contributes to increasing radiation exposure of the patient. ${ }^{30}$ In this work, the number of images acquired was optimized to produce sufficient information for diagnosis. If more images are required with difficult cases, then the dose-area product and effective dose will increase by the same factor when irradiation is directed to the same area of the body. Because the effective dose is dependent on the body area where the radiation-sensitive organs are located, the conversion factors calculated in this study are a useful tool for determining the effective doses of the procedures with a variable number of images.

Previously, a large variation has been observed with the effective doses ranging from 2.1 to $7.4 \mathrm{mSv}$ for cerebral angiography examinations by using DSA. ${ }^{2,10,11,14}$ The effective doses determined in this study were $3.2 \mathrm{mSv}$ (ICRP 60) and 2.7 $\mathrm{mSv}$ (ICRP 103), which are in concordance with those in previous studies. ${ }^{2,10}$ In the present study, the DAP to effectivedose conversion factors for a cerebral DSA examination were 0.065 (ICRP 60) and $0.056 \mathrm{mSv} / \mathrm{Gy}-\mathrm{cm}^{2}$ (ICRP 103), which are somewhat smaller compared with those in previous reports. ${ }^{2,10,11}$ In the present work, the smaller conversion factor for ICRP 103 is a result of the adjustment of the effective dosecalculation scheme between ICRP 60 and ICRP 103; the weight for a high brain dose is higher in ICRP 60 as opposed to ICRP 103. The conversion factors for a cervicocerebral DSA procedure were 0.067 (ICRP 60) and $0.071 \mathrm{mSv} / \mathrm{Gy}^{-\mathrm{cm}^{2}}$ (ICRP 103) and are somewhat higher than those for the cerebral examination, due to the exposure to radiation-sensitive organs in the cervical and thoracic area (ie, thyroid, thymus, sternum, esophagus, and lungs). The conversion factors for cervicocerebral DSA examinations have not been previously published, to our knowledge.

In the assessment of cerebral vessels, the effective doses for CTA were previously reported as 1.9 and $1.6 \mathrm{mSv} .{ }^{12,13}$ In the present study, the corresponding doses were $0.66 \mathrm{mSv}$ (ICRP 60 ) and $0.67 \mathrm{mSv}$ (ICRP 103). The same scanner type and protocol parameters and phantom-dosimeter method were used; only the scan length was $6 \mathrm{~cm}$ longer compared with that in the present study. ${ }^{12}$ This explains the 7-20 times higher absorbed doses for the esophagus and thyroid and the almost 3 times higher effective dose compared with our results. Also a much higher tube voltage (140 versus $120 \mathrm{kV}$ ) was used, and the effective dose was determined by using a previously determined conversion factor. ${ }^{13}$

In the assessment of cervicocerebral vessels, the effective doses for CTA were previously reported as 2.8 and 5.4 mSv. ${ }^{12,13}$ In the present work, the corresponding doses were $4.2 \mathrm{mSv}$ (ICRP 60) and $4.9 \mathrm{mSv}$ (ICRP 103). Cohnen et al ${ }^{12}$ used a shorter cervical scan length and a lower effective tube-current time product compared with the present study. Mnyusiwalla et $\mathrm{al}^{13}$ used the same scan area as in the present study, but the tube voltage was higher and the effective dose was computed by using predetermined conversion factors. An effective dose of 2.2-4.3 mSv for cervicocerebral examinations by using 4 -section to -64 -section CT scanners has been reported. ${ }^{3}$ In general, the effective dose of CTA is strongly dependent on the CT scanner type and the protocol parameters used (ie, the scan length, the tube voltage, and the tube-current time product).

The conversion factors in the present study were 0.0025 $\mathrm{mSv} / \mathrm{mGy}-\mathrm{cm}$ (ICRP 60) and $0.0026 \mathrm{mSv} / \mathrm{mGy}-\mathrm{cm}$ (ICRP 103) for CTA of the cerebral vessels. Both are somewhat higher compared with the conversion factor of $0.0021 \mathrm{mSv} / \mathrm{mGy}-\mathrm{cm}$ reported for CT of the head. ${ }^{13}$ In the present study, the conversion factors for CTA of the cervicocerebral vessels, scanned from the aortic arch to the vertex with constant tube current, were 0.0086 (ICRP 60) and 0.0098 (ICRP 103). These differ from the conversion factor of 0.00345 for a head and neck CT scan, which is calculated as an average of the head and neck conversion factors 0.0021 and $0.0048 .{ }^{13}$ The average value underestimates the effective dose and does not represent the cervicocerebral CTA scan. If the conversion factor from DLP to the effective dose is used with CTA, the scan length and area must be the same as in the setup to determine the conversion 
factor. The reason for this is that the effective dose is in relation to the area of radiation-sensitive organs, and the DLP depends highly on the scan length.

This study reveals that the absorbed doses to the skin, brain, salivary glands, and eyes during diagnostic CTA examinations are lower than those for DSA. Radiation cataract formation has been observed in adults after $\gamma$ irradiation to 100 mGy during childhood. ${ }^{31}$ In a diagnostic procedure, the 100mGy threshold ${ }^{21}$ for brain and skin may be exceeded with children. ${ }^{32}$ The radiation-related injuries must be taken into consideration in the rare cases of imaging infant patients, who often arrive for follow-up studies or require repeated treatments. Also, uncommon radiation-induced hair loss has been reported in patients undergoing several diagnostic DSA and CTA angiography examinations of the brain in a short time interval. ${ }^{7}$ A character of such deterministic effects is that the threshold dose is lower than that when the effects become detectable.

The current setup provides comparable and objective information on the radiation exposure due to CTA and DSA. The coefficient of variation for consecutive simulation runs was under $3.6 \%$ for the effective doses. This is in the range of the overall standard uncertainty in patient dose measurements in diagnostic radiology, which is defined as $\leq 12 \%,{ }^{33}$ and $20 \%$ accuracy is acceptable in cases in which the organ dose is low. ${ }^{34}$ While phantom measurements provide dose data in an average-sized patient, for such a comparative approach, the use of a phantom yields the most comparable data.

\section{Conclusions}

The present study showed that radiation exposure with CTA examinations for the cerebral vessels yields a 5 times lower effective dose for the patient than the same examination performed with DSA. The cervicocerebral CTA protocol causes a one-third higher effective dose compared with the same examination with DSA. This study reveals that the absorbed doses to the skin, brain, salivary glands, and eyes during diagnostic CTA examinations are lower than those for DSA. The conversion factors determined in this study can be used to estimate the effective dose in CTA and DSA of cerebral and cervicocerebral vessels.

\section{References}

1. Teksam M, McKinney A, Cakir B, et al. Multi-slice CT angiography of small cerebral aneurysms: is the direction of aneurysm important in diagnosis? Eur J Radiol 2005;53:454-62

2. Bridcut RR, Murphy E, Workman A, et al. Patient dose from 3D rotational neurovascular studies. Br J Radiol 2007;80:362-66

3. Klingebiel R, Kentenich M, Bauknecht HC, et al. Comparative evaluation of 64-slice CT angiography and digital subtraction angiography in assessing the cervicocranial vasculature. Vasc Health Risk Manag 2008;4:901-07

4. Schuknecht B. Latest techniques in head and neck CT angiography. Neuroradiology 2004;46:208-13

5. McCollough $\mathrm{CH}$, Primak AN, Braun N, et al. Strategies for reducing radiation dose in CT. Radiol Clin North Am 2009;47:27-40

6. Norbash AM, Busick D, Marks MP. Techniques for reducing interventional neuroradiologic skin dose: tube position rotation and supplemental beam filtration. AJNR Am J Neuroradiol 1996;17:41-49

7. Imanishi Y, Fukui A, Niimi H, et al. Radiation-induced temporary hair loss as a radiation damage only occurring in patients who had the combination of MDCT and DSA. Eur Radiol 2005;15:41-46

8. Moritake T, Matsumaru Y, Takigawa T, et al. Dose measurement on both patients and operators during neurointerventional procedures using photoluminescence glass dosimeters. AJNR Am J Neuroradiol 2008;29:1910-17

9. Mamourian AC, Young H, Stiefel MF. Cumulative radiation dose in patients admitted with subarachnoid hemorrhage: a prospective study using a selfdeveloping film badge. AJNR Am J Neuroradiol 2010;31:1787-90

10. Marshall NW, Noble J, Faulkner K. Patient and staff dosimetry in neuroradiological procedures. Br J Radiol 1995;68:495-501

11. McParland BJ. A study of patient radiation doses in interventional radiological procedures. Br J Radiol 1998;71:175-85

12. Cohnen M, Wittsack HJ, Assadi S, et al. Radiation exposure of patients in comprehensive computed tomography of the head in acute stroke. AJNR Am J Neuroradiol 2006;27:1741-45

13. Mnyusiwalla A, Aviv R, Symons S. Radiation dose from multidetector row CT imaging for acute stroke. Neuroradiology 2009;51:635-40

14. Alexander MD, Oliff MC, Olorunsola OG, et al. Patient radiation exposure during diagnostic and therapeutic interventional neuroradiology procedures. J Neurointerv Surg 2010;2:6-10

15. Scalzetti EM, Huda W, Bhatt S, et al. A method to obtain mean organ doses in a Rando phantom. Health Phys 2008;95:242-44

16. Cristy M, Eckerman KF. Specific absorbed fractions of energy at various ages from internal photon sources. I. Methods. Oak Ridge, Tennessee: Oak Ridge National Laboratory; 1987. Report ORNL/TM-8381/V1

17. Kawaura C, Aoyama T, Koyama S, et al. Organ and effective dose evaluation in diagnostic radiology based on in-phantom dose measurements with novel photodiode-dosemeters. Radiat Prot Dosim 2006;118:421-30

18. Ludlow JB, Davies-Ludlow LE, Brooks SL, et al. Dosimetry of 3 CBCT devices for oral and maxillofacial radiology: CB Mercuray, NewTom 3G and i-CAT. Dentomaxillofac Radiol 2006;35:219-26

19. Tapiovaara M, Siiskonen T. PCXMC: a Monte Carlo program for calculating patient doses in medical x-ray examinations. Helsinki, Finland: Radiation and Nuclear Safety Authority; 2008. Report STUK-A231

20. International Commission on Radiologic Protection. ICRP Publication 60: recommendations of the International Commission on Radiological Protection. Annals of the ICRP 1990;21:1-3.

21. International Commission on Radiologic Protection. ICRP 103: 2007 recommendations of the International Commission on Radiological Protection. Annals of the ICRP 2007;37:2-4

22. Manninen AL, Koivula A, Nieminen MT. The applicability of radiophotoluminescence dosimeter (RPLD) for measuring medical radiation (MR) doses. Radiat Prot Dosimetry 2012;151:1-9

23. Brisse HJ, Robilliard M, Savignoni A, et al. Assessment of organ absorbed doses and estimation of effective doses from pediatric anthropomorphic phantom measurements for multi-detector row CT with and without automatic exposure control. Health Phys 2009:303-14

24. Miller DL. Overview of contemporary interventional fluoroscopy procedures. Health Phys 2008;95:638-44

25. Thierry-Chef I, Simon SL, Miller DL. Radiation dose and cancer risk among pediatric patients undergoing interventional neuroradiology procedures. $\mathrm{Pe}$ diatr Radiol 2006;36(suppl 2):159-62

26. Alterio D, Jereczek-Fossa BA, Griseri M, et al. Three-dimensional conformal postoperative radiotherapy in patients with parotid tumors: 10 years' experience at the European Institute of Oncology. Tumori 2011;97:328-34

27. Modan B, Chetrit A, Alfandary E, et al. Increased risk of salivary gland tumors after low-dose irradiation. Laryngoscope 1998;108:1095-97

28. Boukheris H, Ron E, Dores GM, et al. Risk of radiation-related salivary gland carcinomas among survivors of Hodgkin lymphoma: a population-based analysis. Cancer 2008;113:3153-59

29. Barrett JF, Keat N. Artifacts in CT: recognition and avoidance. Radiographics 2004;24:1679-91

30. Miller DL, Balter S, Noonan PT, et al. Minimizing radiation-induced skin injury in interventional radiology procedures. Radiology 2002;225:329-36

31. Wilde G, Sjöstrand J. A clinical study of radiation cataract formation in adult life following gamma irradiation of the lens in early childhood. $\mathrm{Br}$ J Ophthalmol 1997;81:261-66

32. Glennic D, Connolly B, Gordon C. Entrance skin dose measured with MOSFETs in children undergoing interventional radiology procedures. Pediatr Radiol 2008;38:1180-87

33. Zoetelief J, Julius HW, Christensen P. Recommendations for patient dosimetry in diagnostic radiology using TLD. Brussels, Belgium: Office for Official Publications of the European Communities;2000. Report No EUR 196042000

34. Pernička F, McLean ID. Dosimetry in Diagnostic Radiology: An International Code of Practice. Vienna, Austria: International Atomic Energy Agency; 2007. Report No TRS 457 